\title{
La enfermedad de Chagas congenita en la Provincia de Salta, Argentina, años 1980-1997
}

\author{
Congenital Chagas' disease in the Province of Salta, \\ Argentina, from 1980 to 1997
}

Mario Zaidenberg

\begin{abstract}
Resumen Se presenta la experiencia de 18 años en la provincia de Salta en el manejo de recién nacidos con enfermedad de Chagas congénita. Desde distintos ámbitos del sistema provincial de salud, el Hospital Materno-infantil de la ciudad de Salta, hospitales del interior y la atención ambulatoria se detectaron y diagnosticaron 102 recién nacidos $(R N)$ y lactantes con infección congénita. Los RN se dividieron en dos grupos mayores, el último subdivido, de acuerdo a la oportunidad diagnóstica. Se describe la metodología diagnóstica, presentación clínica, tratamiento y el seguimiento posterior de los niños tratados. Se analizan las características de la experiencia y se discuten las condiciones específicas del diagnóstico, tratamiento y seguimiento de los niños estudiados. Se describen las recomendaciones empleadas en la provincia en el programa de control de Chagas perinatal así como las conclusiones derivadas de esta experiencia.
\end{abstract}

Palabras-claves: Enfermedad de Chagas congénita. Infección perinatal por Trypanosoma cruzi.

\begin{abstract}
This is the experience of 18 years of attending newborn infants suffering from congenital Chagas' disease. From a variety of sources in the provincial health system, the Mother-Child Hospital in the city of Salta, country hospitals and out-patient care, 102 newborn and infants were detected and diagnosed. The newborn were classified into two main groups, one being subclassified according to diagnostic opportunity. The diagnostic methodology, clinical presentation, treatment and follow-up of the children under care are described, the characteristics of the experience analyzed and the specific conditions of the diagnostic discussed. Also described are the recommendations used in the province in the perinatal Chagas control program, as well as the conclusions reached by this experience.
\end{abstract}

Key-words: Congenital Chagas' disease. Perinatal infection by Trypanosoma cruzi.

La enfermedad de Chagas congénita constituye por si misma un problema de salud pública 2 y es una vía de infección que constituye uno de los desafíos de la lucha contra la endemia chagásica en América latina. Los diferentes países del Cono sur poseen ya indicadores de infestación por triatomíneos que evidencian en el corto plazo que la vía vectorial de la transmisión por el Trypanosoma cruzino significará un peligro real para la aparición de casos nuevos de Chagas aguda, de mantenerse y consolidarse la vigilancia entomológica emprendida ${ }^{14}$.

Esta afirmación es particularmente cierta en regiones donde se ha alcanzado un aceptable nivel de control de la infestación domiciliaria y el control de la sangre a transfundir. Tal es el caso de la provincia de Salta, donde desde hace aproximadamente una década se mantiene una

Servicio Nacional de Chagas, Salta, Argentina.

Endereço para correspondencia: Dr. Mario Zaidenberg. Servicio Nacional de Chagas. Gral. Güemes 125, 1ำ Piso, Casilla Postal 4400 Salta, República Argentina.

Fax: 0054387 431-0684/421-0630.

Recebido para publicação em 14/9/98. 
infestación domiciliaria promedio que oscila entre 3 y 1,6\%, (Informe del Programa Provincial de Chagas, Ministerio de Salud Pública Salta, 1997).

Además los bancos de sangre existentes en el ámbito oficial controlan sistemáticamente la sangre a transfundir, lo que disminuye sensiblemente las posibilidades de adquirir la infección por esta vía.

La enfermedad de Chagas congénita en nuestro medio presentó una incidencia de $4 \%$ de acuerdo al único estudio de detección sistemática de la infección realizado en embarazadas ${ }^{19}$. La prevalencia de infección chagásica en embarazadas en este período osciló entre 165\% 16 y $10 \%$ (informe Gerencia sanitaria del Hospital Materno-Infantil, HMI, 1996).
El objetivo de este trabajo es describir las características clínicas, diagnósticas y terapéuticas de los recién nacidos (RN) y lactantes con enfermedad de Chagas congénita detectados entre los años 1980-1997. La experiencia refleja algunos de estos aspectos en diferentes instancias: 1) la asistencial recogida en el Servicio de Neonatología del HMI de Salta; 2) la detección ambulatoria de lactantes infectados menores de 2 años derivados al Servicio Nacional de Chagas, Salta y los egresados de la unidad de lactantes del HMI entre los años 1995-1996; y 3) la etapa lleva a cabo mediante la estrategia de atención Primaria de la salud entre los años 1987-1988 en cuatro áreas operativas de la provincia: Salta capital, Orán, Tartagal y General Güemes.

\section{MATERIAL Y METODOS}

Diagnóstico. Embarazadas: las pruebas serológicas utilizadas fueron la hemaglutinación indirecta (HAI), título 1/16 o superior, prueba de tamizaje, y como pruebas confirmatorias de infección, la inmunofluorescencia indirecta (IFI), igual título, o ELISA (Enzyme Linked ImmuneSorbent Assay) cualitativa. En todos los casos se consideró infección cuando las dos pruebas serológicas fueron reactivas, HAI e IFI o HAI y ELISA.

Recién nacidos (RN) y lactantes menores de 2 años: Se usaron la gota fresca en los primeros casos y sistemáticamente el método del micro-hematócrito ${ }^{9} 18$ hasta los seis meses de vida. El método confirmatorio de infección en esta etapa fue el parasitológico, mediante la visualización directa del Trypanosoma cruzi. A los primeros quince casos diagnosticados en le curso de los primeros dos meses de vida se les realizaron pruebas de aglutinación directa con y sin 2 mercaptoetanol $^{219}$. Luego del sexto mes el criterio de infección fue la detección de pruebas serológicas reactivas.

La capacitación y evaluación de los $\mathrm{RN}$ y lactantes infectados tuvo dos instancias operativas:

Servicio de Neonatología: con la excepción de los primeros doce casos diagnosticados, en que todas las embarazadas contaron con pruebas serológicas, y posteriormente los $\mathrm{RN}$ hijos de madres chagásicas; en el resto se solicitaron cuando la madre tenía serología reactiva o ante la sospecha clínica de infección intrauterina cuando no se contaba con la serología materna.

Ambulatoria: Un grupo de lactantes fue detectado en un consultorio externo del área
Capital producto de la derivación de lactantes hijos de madres chagásicas.

Otro grupo de RN y lactantes fue detectado por el equipo de APS entre los años 1987-1988. La experiencia consistió en un programa de control de Chagas perinatal en cuatro áreas operativas(regiones sanitarias cubiertas por personal de APS). Orán, Gral Güemes, Tartagal, y Salta capital. El mismo incluyó la detección de las embarazadas y la realización de pruebas serológicas para Chagas y seguimiento en los consultorios externos de los hospitales de igual forma loa agentes sanitarios lo hicieron durante las visitas domiciliarias hasta el momento del parto. Posteriormente se realizó el seguimiento de los RN hasta los seis meses de vida, en que se efectuaba el último control serológico.

Los RN y lactantes diagnosticados se dividieron de acuerdo a la etapa diagnóstica en:

- Grupo I, diagnosticados entres del mes de vida;

- Grupo lla diagnosticado entre el primer y sexto mes y

- Grupo llb, entre el séptimo y los 24 meses.

Todos los RN fueron evaluados clínicamente, pesados y la edad gestacional evaluada por el puntaje de Capurro ${ }^{6}$. Los lactantes mayores de un mes fueron evaluados mediante la recolección de antecedentes, examen físico y análisis clínicos de laboratorio. Se estableció una comparación de la expresión clínica entre los $\mathrm{RN}$ y lactantes del los Grupos I y II mediante una tabla de contingencia de acuerdo al presencia de uno 0 ningún signo y por otro, dos o más signos atribuibles a infección chagásica; se usó la 
prueba del ji². La hipótesis nula estableció que no había diferencias en la expresión clínica entre los grupos de acuerdo a la etapa diagnóstica. Se comparó en la Tabla 1 mediante una Tabla de $r \times c$, en la que se compararon cada uno de los signos por grupos y los asintomáticos. En la Tabla 2 se compararon los asintomáticos versus los $\mathrm{RN}$ y lactantes con uno o más signos, en ambas comparaciones se empleó la prueba del ji².

Se realizaron exámenes clínicos del laboratorio que incluyeron hemograma, glucemia, uremia, eritrosedimentación, parcial e orina, dosaje de transaminasas oxalo-acética y glutámico-pirúvica. Además de acuerdo a la condición clínica de los RN se solicitaba electrocardiogramas, ecocardiografías, ecografías cerebrales, exámenes citoquímico y bacteriológicos del líquido cefalo-raquídeo, etc.

Una vez confirmada la infección se inició el tratamiento con benznidazol®. Los primeros 12
$\mathrm{RN}$ diagnosticados se trataron a $7 \mathrm{mg} / \mathrm{kg} / \mathrm{día}$. El resto fue tratado a razón de $5 \mathrm{mg} / \mathrm{kg} /$ día distribuidos en dos dosis diarias. Se realizaron exámenes clínicos semanales, control de peso y talla, observación de aparición de efectos adversos, y análisis de laboratorio que incluyeron hemograma, uremia, dosaje de transaminasas, parcial de orina al inicio, mitad y final del tratamiento.

Los RN diagnosticados en el primer mes y tratados específicamente fueron controlados con pruebas serológicas cada 4-6 meses e incluso anualmente hasta su negativización. Aquellos lactantes infectados hijos de madres chagásicas no diagnosticados en el primer mes, se les practicaron exámenes parasitológicos y pruebas serológicas hasta el sexto mes de vida; o se detectaron en encuestas serológicas sistemáticas cuando aún eran menores de 2 años. El seguimiento posterior fue similar a los primeros.

Tabla I - Expresión clínica de los RN y lactantes procedentes del Grupo I y Grupo lla y b.

\begin{tabular}{|c|c|c|c|c|c|c|c|c|}
\hline \multirow[b]{3}{*}{ Signos } & \multicolumn{8}{|c|}{ Grupos } \\
\hline & \multicolumn{2}{|c|}{$\mathrm{I}(\mathrm{n}=71)$} & \multicolumn{2}{|c|}{ lia $(n=14)$} & \multicolumn{2}{|c|}{$\operatorname{lib}(\mathrm{n}=17)$} & \multicolumn{2}{|c|}{ Total $(n=102)$} \\
\hline & no & $\%$ & $\mathrm{n}$ o & $\%$ & $\mathrm{n}^{\circ}$ & $\%$ & $\mathrm{n}^{0}$ & $\%$ \\
\hline Hepatomegalia & 48 & 67,6 & 7 & 50,0 & 5 & 29,4 & 60 & 58,8 \\
\hline Esplenomegalia & 37 & 52,1 & 4 & 28,6 & 2 & 11,8 & 43 & 42,1 \\
\hline Ictericia & 40 & 56,3 & 1 & 7,1 & 0 & 0,0 & 41 & 40,2 \\
\hline Anemia & 37 & 52,1 & 2 & 14,3 & 1 & 5,9 & 40 & 39,2 \\
\hline Prematurez & 26 & 36,6 & 2 & 14,3 & 1 & 5,9 & 29 & 28,4 \\
\hline Hidrops fetalis & 5 & 7,0 & 0 & 0,0 & 0 & 0,0 & 5 & 4,9 \\
\hline Meningo-encefalitis & 4 & 5,6 & 0 & 0,0 & 0 & 0,0 & 4 & 3,9 \\
\hline Asintomático & 15 & 21,1 & 7 & 50,0 & 12 & 70,6 & 34 & 33,3 \\
\hline
\end{tabular}

ji ${ }^{2}$ para Tabla $\mathrm{r} \times \mathrm{c}, 0$ 51,57, $\mathrm{p}=0,00000$. Al comparar por estratos y si se excluyen hidrops fetalis y meningo-encefalitis (propios de Grupo I), $p=0,03$.

Tabla 2 - Expresión cuantitativa de los signos de infección de acuerdo a etapa diagnóstica.

\begin{tabular}{|c|c|c|c|c|c|c|c|c|}
\hline \multirow[b]{3}{*}{ № signos } & \multicolumn{8}{|c|}{ Procedencia } \\
\hline & \multicolumn{2}{|c|}{$\mathrm{I}(\mathrm{n}=71)$} & \multicolumn{2}{|c|}{ lia $(n=14)$} & \multicolumn{2}{|c|}{ lib $(n=17)$} & \multicolumn{2}{|c|}{ Total $(n=102)$} \\
\hline & $\mathrm{n}^{\circ}$ & $\%$ & $\mathrm{n}^{0}$ & $\%$ & $\mathrm{n}^{\circ}$ & $\%$ & $\mathrm{n}^{0}$ & $\%$ \\
\hline Asintomáticos & 15 & 21,1 & 7 & 50,0 & 12 & 70,6 & 34 & 33,3 \\
\hline 1 & 6 & 8,5 & 2 & 14,3 & 2 & 11,8 & 10 & 9,8 \\
\hline 2 & 9 & 12,7 & 2 & 14,3 & 2 & 11,8 & 13 & 12,7 \\
\hline 3 & 11 & 15,5 & 2 & 14,3 & 1 & 5,9 & 14 & 13,7 \\
\hline 4 & 14 & 19,7 & 1 & 7,1 & 0 & 0,0 & 15 & 14,7 \\
\hline 5 & 11 & 12,7 & 0 & 0,0 & 0 & 0,0 & 11 & 10,8 \\
\hline 6 & 5 & 7,0 & 0 & 0,0 & 0 & 0,0 & 5 & 4,9 \\
\hline
\end{tabular}

$\mathrm{ji}^{2}=17,13, \mathrm{p}=0,00019$

\section{RESULTADOS}

Durante el período 1980 - 1997 se diagnosticaron 102 recién nacidos y lactantes con enfermedad de Chagas congénita. De acuerdo al momento diagnóstico se dividieron a los $\mathrm{RN}$ y 
lactantes en dos grupos: Grupo I: $(n=71)$ diagnosticados antes de cumplir el primer mes de vida. Se diagnosticaron 64 en el HMl y 7 mediante la estrategia ambulatoria. En todos los casos el parto fue institucional; siete procedieron de otros servicios; Grupo II $(n=31)$, diagnosticados entre el primer mes y los 24 meses. Se subdividió en: Ila, $n=14$; (cinco en el HMI y 9 ambulatorios) diagnosticados entre los 2 y 6 meses de vida que incluyeron 7 en el segundo, 4 en el cuarto y 3 en el sexto mes y llb, $n=17$, (cuatro en el HMl y 13 ambulatorios) cuyo diagnóstico se realizó entre los 7 y 24 meses en la consulta externa: 5 a los 12 meses, 3 a los 18 y 3 a los 21 meses.

Diagnóstico. Grupo I $(\mathbf{n}=\mathbf{7 1})$. En todos los casos se realizó con el método del microhematócrito y la gota fresca sólo se usó en los primeros casos 17, 18.

Los títulos de las pruebas serológicas de los $\mathrm{RN}$ se correspondieron con los títulos maternos, con la excepción de $3 \mathrm{RN}$ en los cuales fueron superiores. El rango para la HAl fue de $1 / 32$ y $1 /$ 512 y la IFI fue de 1/16 a 1/1024. Se observó una disminución progresiva de los títulos hasta alcanzar la negativización entre los 4 y 8 meses. Una excepción fue el caso No. 4, caracterizado como fracaso terapéutico. Fue tratado con nifurtimox durante 60 días y alcanzó la negativización de las pruebas serológicas a los 14 meses 18. Con posterioridad al primer año de seguimiento, los controles serológicos se realizaron en forma semestral e incluso anual cuando la localización de los pacientes era dificultosa.

Grupo II $(n=31)$ a. $(n=14)$. El diagnóstico se realizó con el micro-hematócrito. b. $(\mathbf{n}=17)$. Se diagnosticaron con el par serológico convencional, HAI-IFI o HAI-ELISA.

Clínica. Ver Tablas 1 y 2 . La edad gestacional varió entre 26 y 40 semanas. Veinte $R N$ pretérmino fueron $A E G$ y $P E G$. El rango del peso varió entre 750 y $3700 \mathrm{~g}$; el promedio fue de $2750 \mathrm{~g}$.

La hepatomegalia $(=0+$ de $2 \mathrm{~cm}$ por debajo del reborde costal) se detectó en el nacimiento o en los primeros días de vida y fue detectable entre los 2 y 3 meses.

La esplenomegalia(ídem hepatomegalia), persistió ente 2 y 4 meses.

La anemia $(\mathrm{Hb}$ inferior a $8 \mathrm{~g} \%$ y hematócrito menor de $30 \%$ ), se mantuvo entre 3 a 5 meses, los RN de más bajo peso presentaron una tendencia a recuperar valores normales más tardíamente.
La ictericia apareció al $2^{\circ}-3^{\circ}$ día y persistió entre la segunda y tercera semana de vida.

Estos signos generalmente se presentaron con el nacimiento o inmediatamente después. La mayoría de los $\mathrm{RN}$ presentaron una combinación de estos signos; la mayor prevalencia de compromiso orgánico se dio en los $\mathrm{RN}$ prematuros.

Los cuadros de edema generalizado se presentaron en $4 \mathrm{RN}$ prematuros de 27-28 semanas y 1 RNT de 37 semanas de gestación. El edema fue generalizado, la temperatura conservada y no presentaron signo de godet. En un $\mathrm{RN}$ persistió 3 semanas, otro $\mathrm{RN}$ sufrió una sobreinfección bacteriana y falleció por sepsis a Pseudomonas aeruginosa a las 6 semanas. La meningoencefalitis (presencia de Trypanosoma cruzi en LCR) se presentó en 1 RN pretérmino y tres de término. De estos, 3 no presentaron sintomatología neurológica y evolucionaron sin complicaciones. El cuarto RN, de término, fue una co-infección no confirmada por carga viral o autopsia, que al nacer presentó una hepatoesplenombegalia, edema generalizado, síndrome de dificultad respiratoria y síndrome purpúrico. Se practicaron diversos cultivos, incluyendo LCR, cuyo examen citoquímico fue normal. Se medicó con antibióticos, y se mantuvo con medidas de sostén respiratorio e hidratación parenteral. Evolucionó de manera errática hasta que en el día 20 sufrió un síndrome convulsivo, por lo que fue punzado y al examen directo del LCR fueron observados Trypanosoma cruzi circulantes. Se medicó con benznidazol, sin embargo continuó con convulsiones subintrantes con agravamiento del estado general y posterior fallecimiento a los 28 días de vida.

El resto de los $\mathrm{RN}$ presentó una combinación de diversos trastornos no vinculados a la enfermedad de Chagas congénita: sufrimiento fetal agudo, asfixia neonatal, depresión neonatal leve, sepsis bacteriana, síndromes de dificultad respiratoria leves a moderados, diarreas y complicaciones metabólicas, Tabla 3.

Laboratorio clínico. Los análisis clínicos obtenidos en los primeros $12 \mathrm{RN}$ fueron motivo de una publicación ${ }^{18}$.

Grupo I. La anemia se caracterizó como hipocrómica y microcítica. La hiperbilirrubinemia fue a predominio de bilirrubina indirecta en 29 casos, cinco casos presentaron aumento de la bilirrubina directa que coincidieron con un aumento de las transaminasas. Las transaminasas aumentaron ente $40-125 \mathrm{~m} / \mathrm{ul} / \mathrm{ml}$ y las TGP entre 
Tabla 3 - Comparación de la expresión clínica entre los Grupos I y II.

\begin{tabular}{lccc}
\hline No. Signos & Grupo I & Grupo II & Total \\
\hline$=0>1$ & 21 & 23 & 44 \\
$2 y+$ & 50 & 8 & 58 \\
\hline Total & 71 & 31 & 102 \\
\hline
\end{tabular}

$\mathrm{Ji}^{2}$, Mantel-Haenzel 17,34, $\mathrm{p}=0,00003$

45 y $150 \mathrm{mul} / \mathrm{ml}$ en $25 \mathrm{RN}$ (cinco de estos $\sin$ hiperbilirrubinemia). Regresaron a los valores normales entre los 30 y 60 días.

El examen del LCR de uno de los $3 \mathrm{RN}$ con Trypanosoma cruzi reveló pleocitosis linfocitaria (200 elementos $/ \mathrm{mm}^{3}$ ) y aumento en la concentración de albúmina, el restante, con co-infección por el $\mathrm{VIH}$, presentó 30 linfocitos por $\mathrm{mm}^{3}$, xantocrómico, glucorraquia normal, Pandy (+).

Grupo Ila. Dos RN con hepatomegalia presentaron elevación de las TGP-TGO y 1 , anemia microcítica e hipocrómica. Los exámenes de laboratorio en el resto de los lactantes de este grupo fueron normales.

Ilb. Un RN presentó anemia con características similares a los anteriores. El resto de los análisis fue normal.
Seguimiento serológico. Los RN del grupo I fueron seguidos entre 9 y 12 años, con un número variable de lactantes y niños recuperados. Antes del año de seguimiento, 66/69 negativizaron sus pruebas serológicas. Entre los 5-8 años, se detectaron 35 , de los que 32 fueron no reactivos. En el período de 9 y 12 años se controlaron 24 niños de los que 22 mantuvieron la seronegatividad de sus reacciones, Tabla 4. Los lactantes del grupo II se controlaron en un período menor de acuerdo al breve lapso transcurrido desde su diagnóstico, Tabla 4.

Tratamiento. El tratamiento se administró entre 30 y 42 días. Siete presentaron deposiciones diarreicas, 6 distensión abdominal y 3 erupción dérmica. En dos casos se suspendió el tratamiento y se reinició con dosis más bajas. Tres $\mathrm{RN}$ presentaron detención del crecimiento. Luego de

Tabla 4 - Seguimiento serológico de 69 RN del Grupo I y 28 lactantes del Grupo II durante 12 años. Salta, 1980-1997.

\begin{tabular}{|c|c|c|c|c|c|c|c|c|c|c|c|c|c|c|c|}
\hline \multirow{3}{*}{$\begin{array}{l}\text { Años } \\
\text { Dx Resul }\end{array}$} & \multicolumn{3}{|c|}{$>1$} & \multicolumn{3}{|c|}{1} & \multicolumn{3}{|c|}{ 2-4 } & \multicolumn{3}{|c|}{$5-8$} & \multicolumn{3}{|c|}{$9-12$} \\
\hline & \multirow[t]{2}{*}{$E$} & \multicolumn{2}{|c|}{ NR } & \multirow[t]{2}{*}{ E } & \multicolumn{2}{|c|}{$\mathrm{NR}$} & \multirow[t]{2}{*}{ E } & \multicolumn{2}{|c|}{ NR } & \multirow[t]{2}{*}{$E$} & \multicolumn{2}{|c|}{ NR } & \multirow[t]{2}{*}{$E$} & \multicolumn{2}{|c|}{ NR } \\
\hline & & $\mathrm{F}$ & $\%$ & & $\mathrm{~F}$ & $\%$ & & $\mathrm{~F}$ & $\%$ & & $\mathrm{~F}$ & $\%$ & & $\mathrm{~F}$ & $\%$ \\
\hline Grupo I & 69 & 66 & 95,7 & 61 & 55 & 90,2 & 43 & 39 & 90,7 & 35 & 32 & 91,4 & 24 & 22 & 91,7 \\
\hline Grupo lia & & & & 13 & 12 & 92,3 & 11 & 10 & 90,9 & & & & & & \\
\hline Grupo lib & & & & & & & 15 & 13 & 86,7 & & & & & & \\
\hline
\end{tabular}

Años: etapa diagnóstica; Dx Resul: resultados diagnósticos; E: estudiados; NR: no reactivos; F: frecuencia; \%: porcentaje de no reactivos.

finalizar el mismo, recuperaron el peso perdido. Los controles de laboratorio no se modificaron durante el tratamiento. Las TGO-TGP que estaban aumentadas al iniciarlo, continuaron con valores similares y alcanzaron valores normales entre los 30 y 45 días de finalizar el tratamiento.

\section{DISCUSIÓN}

La enfermedad de Chagas congénita en nuestro medio se manifestó con la expresión de un signo como hepatomegalia, esplenomegalia, anemia aisladamente o por la combinación de los 4 o 5 signos más frecuentes: hepatomegalia, esplenomegalia, anemia ictericia y prematurez. El edema generalizado o la meningoencefalitis fueron de presentación infrecuente. Esta última entidad, tuvo similares características a las descriptas por Howard ${ }^{10}$, en el sentido de no presentar una manifestación clínica específica ${ }^{18}$.
Los signos clínicos se presentaron solos o asociados; también fue común la presentación simultánea de signos y síntomas inespecíficos con relación a la infección chagásica congénita. Del total de $\mathrm{RN}$ y lactantes diagnosticados el $43,1 \%$ en esta experiencia tuvo uno o ningún signo atribuible a la infección. $Y$ aún cuando estuvieran asociados al resto de los signos, como prematurez, ictericia, anemia, no comprometieron por si mismos la sobrevida de los RN. Los únicos decesos de esta experiencia, fueron dos: un 
prematuro de muy baja edad gestacional que en curso de su internación adquirió una infección intrahospitalaria por germen Gram negativo y el otro RN, se trató de una co-infección por el VIH con compromiso meningoencefalítico severo. La mayor parte de esta experiencia se llevó a cabo en el Hospital Materno-Infantil de la ciudad de Salta, que es el centro de referencia obstétrico neonatal de la provincia. Por lo tanto en sus servicios son asistidos embarazos y $\mathrm{RN}$ de alto riesgo, con una alta prevalencia de enfermedades neonatales que modifican o complican la morbilidad atribuible a la infección chagásica. No obstante, esta proporción supera el $10 \%$ de la población de RN prematuros de la institución. Por lo cual, podría explicar parcialmente la alta proporción de prematuros, $36,6 \%$ en el grupo I y $28,4 \%$ en todos los RN y lactantes estudiados, así como de anemia e ictericia, complicaciones frecuentes de esta categoría de RN. Más aún, la presentación de prematurez es ampliamente superior al de otras experiencias, como la Freilij en Buenos Aires con 13\% 6 o Bittencourt en Bahía con $10,5 \%{ }^{5}$.

La etapa neonatal continúa siendo el período donde se realiza el mayor número de diagnósticos como ya fue establecido 8,1 . Sin duda, a este hecho contribuyeron la presentación simultánea de varios signos clínicos que orientaron el diagnóstico. En este sentido fue clara la diferencia establecida al comparar la expresión clínica de los $R N$ versus lactantes de 1 a 24 meses. En el Servicio de Neonatología facilitó el diagnóstico la identificación previa de la serología reactiva en la madre, lo que orientó los estudios y el seguimiento de los $\mathrm{RN}$; por otro lado, la presentación simultánea de varios signos provocó que el equipo de salud del área tuviera ante la presentación del síndrome cuyo acrónimo es Storch (sífilis, toxoplasmosis, rubéola, citomegalovirus, herpes y Chagas en nuestro medio), una dedicación más focalizada en la detección de la enfermedad de Chagas congénita entre otras.

En los últimos años, una mayor difusión de las actividades de control del Chagas perinatal en el equipo de salud posibilitó que un número creciente de $\mathrm{RN}$ con enfermedad de Chagas congénita sean diagnosticados (Informe Programa de Chagas, Epidemiología, MSP. 1994). La metodología consiste básicamente en:

1. La implementación sistemática de controles serológicos en las embarazadas. Por lo tanto, el equipo de salud que desarrolla tareas en hospitales o centros de salud de mediana y baja complejidad en la población general, sin el sesgo de un hospital de referencia, puede contar con un elemento objetivo de sospecha como una prueba serológica reactiva para la detección de y/o seguimiento del hijo de madre chagásica. Por otro lado, se ha demostrado que la seropositividad de las mismas está asociada con un exceso de riesgo que sus niños sean también seropositivos 15 , y

2. El control y seguimiento parasitológico y serológico de los RN hijos de madres chagásicas hasta los seis meses por lo menos.

El perfil clínico de los RN diagnosticados en esta experiencia, reveló que la morbilidad por la enfermedad de Chagas congénita es leve en términos generales, tal como se afirmó. Por lo cual, en nuestro medio el diagnóstico del RN infectado no tiene la urgencia que eventualmente pueden tener otras entidades clínicas en esta etapa como la sífilis, toxoplasmosis, herpes, citomegalovirus ${ }^{1}$. Por tanto, no parece justificado por el momento el empleo de herramientas diagnósticas de alta sensibilidad como los antígenos recombinantes, $\mathrm{PCR}^{7114}$, pero de alto costo y complejidad con relación a los potenciales beneficios de su empleo en un medio que por lo general debe manejarse con recursos precarios y personal no entrenado en dichas técnicas ${ }^{17}$. En este sentido, tanto el micro-hematócrito en los primeros meses de vida ha demostrado una sensibilidad superior al $90 \%$ en nuestro medio ${ }^{19}$, un valor predictivo positivo de $100 \%$ y un valor predictivo negativo de $99,7 \%$ en la experiencia del Hospital de Niños de Buenos Aires ${ }^{8}$. Luego, el empleo de la serología convencional de acuerdo a las normas establecidas $^{12}$, posee una alta seguridad en el diagnóstico y su manejo técnico no ofrece dificultades para el personal de salud.

En relación al seguimiento de los $\mathrm{RN}$ del Grupo I tratados, se pudo observar entre los 5 a 8 años de seguimiento, con una recuperación de aproximadamente $50 \%$ de la población estudiada, que el $91,4 \%$ de los niños controlados mantuvieron la negativización de las pruebas serológicas realizadas, estas proporciones se mantuvieron en los seguimientos más alejados, entre 9 a 12 años.

El Grupo II, con un diagnóstico más tardío y con períodos de seguimiento menores presentó un porcentaje inferior de serología no reactiva. Probablemente al haberse tratado más tardíamente con relación a los $\mathrm{RN}$, la reversión de los título serológicos reactivos necesite mayor tiempo para que tenga lugar tal efecto. 
La enfermedad de Chagas congénita, aún cuando desconocemos delicados mecanismos fisiopatogénicos y eventualmente factores de riesgo asociados a la transmisión materno-fetal ${ }^{1}$, es una entidad clínica que debería incluirse dentro del grupo de enfermedades perfectamente controlables en los primeros años de vida. A nivel operativo se cuenta con elementos suficientemente probados para tratar en forma oportuna y eficaz esta dolencia. Sólo con una decisión sanitaria fuerte y consistente se podrá lograr tal objetivo.

\section{AGRADECIMIENTOS}

El autor agradece al personal del Hospital Materno-Infantil, centros de salud de la ciudad de Salta, hospitales de Orán, Tartagal, Gral. Güemes, y es especial a los agentes sanitarios y enfermeras, quienes desde diferentes lugares de la provincia de Salta, contribuyeron para la realización y conocimiento de este trabajo.

\section{REFERENCIAS BIBLIOGRAFICAS}

1. Andrade ALSS. Epidemiologic research in congenital $T$. Cruzi infection. Memórias do Instituto Oswaldo Cruz 89(supl I):32, 1994.

2. Andrade ALSS, Zicker F, Silva IG, Souza JMP, Martelli CMT. Risk factors for Trypanosoma cruzi infection among children en Central Brazil. A case-control study in vector control settings. The American Journal of Tropical Medicine and Hygiene 52:183-187, 1995.

3. Azogue E, Darras C. Estudio prospectivo de la enfermedad de Chagas en Recién nacidos con infección placentaria por Trypanosoma cruzi (Santa Cruz-Bolivia). Revista da Sociedade Brasileira de Medicina Tropical 24:105-109,1991.

4. Bittencourt AL. Doença de Chagas congênita como un problema de Saúde pública. Annales Societé Belge Médicine Tropicale 65(supl 1):103-106, 1985.

5. Bittencourt AL, Barbosa HS, Rocha T, Santos I, Sodré A. Incidência da transmissão congênita da doença de Chagas em partos prematuros na Maternidade Tsylla Balbino (Salvador, Bahia). Revista do Instituto de Medicina Tropical de São Paulo 14:131-134, 1972.

6. Capurro H. A simplified method for diagnosis of gestational age in the newborn infant. Jornal de Pediatria 93:120-122, 1978.

7. Frasch ACC, Reyes MB. Diagnosis of Chagas' disease using recombinant DNA technology. Parasitology Today 6:137-139, 1990.

8. Freilij H, Altchech J. Congenital Chagas' disease: diagnostic and clinical aspects. Clinical Infectious Diseases 21:551-555, 1995.

9. Freilij J, Muller L, Cappa GS. Direct micromethod for diagnosis of acute and congenital Chagas' disease. Journal of Clinical Microbiology 18:327-330, 1993.

10. Howard J. Clinical aspects of congenital Chagas' disease. In new approaches for American trypanosomiasis research. Scientific Publication $n \div 318$. Washington, PAHO/WHO, p. 212-215, 1975.

11. Lorca M. Congenital Chagas' disease: Serological diagnosis trough conventional techniques and methods of molecular biology. RT-005. Memórias do Instituto Oswaldo Cruz 89(supl I):30, 1994.

12. Ministerio de Salud y Acción Social de la Nación, Secretaria de Programas de Salud. Manual de atención del pacinte infectado chagásico. Buenos Aires, p. 8, 1997.

13. Organización Panamericana de la Salud-Organización Mundial de la Salud. PNPS/92-18 revisión 1. Iniciativa del Cono Sur Agosto 1991-diciembre 1992. Estado actual de las acciones para la eliminación de la transmisión vectorial e interrupción de la transmisión transfusional del Trypanosoma cruzi, 1992.

14. Reyes MB, Lorca P, Muñoz P, Frasch ACC. Fetal specificities against Trypanosoma cruzi infected newborns. Medical Sciences. Proceedings of the National Academy Sciences 87:2844-2850, 1990.

15. Sánchez JP, Siegel JD. Infecciones congénitas y perinatales. Grupo herpes. Citomegalovirus. Virus herpes simple. Sífilis. Toxoplasmosis. In:Oski FA, De Angelis C, Feigin RD, Warshaw JB (eds) Pediatría. Principios y práctica. Editorial Médica Panamericana SA, 1993.

16. Vattuone NH, Yanovsky JF. Trypanosoma cruzi: Agglutination activity of enzyme-treated epimastigotes. Experimental Parasitology 30:359-63, 1971.

17. Zaidenberg M. Meningoencefalitis chagásica en Recién nacidos, 1980-1997, Salta. In: Resumenes del $2^{\circ}$ Congreso Argentino de Zoonosis, $1^{\circ}$ Congreso Argentino y Latinoamericano de Enfermedades Emergentes, Buenos Aires, p. 216, 1998.

18. Zaidenberg M. Enfermedad de Chagas congénita. Temas de zoonosis y enfermedades emergentes. In:Resumenes del $2^{\circ}$ Congreso Argentino de Zoonosis, Asociación Argentina de Zoonosis, Buenos Aires. p. 114-148, 1998.

19. Zaidenberg M, Segovia A. La enfermedad de Chagas congénita en la ciudad de Salta, Argentina. Revista do Instituto de Medicina Tropical de São Paulo 10:35-43, 1993.

20. Zaidenberg MO, Segovia MA. Prevalencia de la infección chagásica en embarazadas asistidas en el hospital de Maternidad e Infancia, Salta. In: Resumenes del $2^{\circ}$ Congreso Argentino de Protozoología, p. 71, 1984. 